\title{
PROPAGATION OF STRESS PULSES IN A PERIODICALLY LAYERED ELASTIC COMPOSITE
}

\author{
A. K. Grosh \\ Reactor Analysis and Studies Section, Bhabha Atomic Research Centre, Bombay, India \\ S. C. LAKKAD \\ Department Aeronautical Engineering, \\ AND \\ P. RAMAKRISHNAN \\ Department Metallurgical Engineering, Indian Institute of Technology, Bombay, India
}

\begin{abstract}
A transfer matrix analysis of a periodically layered elastic composite is presented. Numerical analysis of propagation of a rectangular stress pulse reveals attenuation and development of a stress of an opposite sign, and these results are shown as functions of location and pulse-width.
\end{abstract}

\section{INTRODUCTION}

Attenuation of stress pulses in a periodically layered elastic composite is a parameter of design interest for dynamic loading. Earlier workers have studied this either by a transient analysis or by devising methods to arrive directly at the attenuation within the framework of a one-dimensional theory. Sve [1] has presented a transient analysis for step stress input by assuming a spatial wave form and using the dispersion relation for wave number as a function of frequency. Mukoniki and Ting [2] have also presented a similar analysis for a step-stress input. However, both these analyses are based on Floquet's quasi-periodic relation limiting the type of boundary conditions [3]. Essentially these studies are, therefore, limited to semi-infinite media. Christensen [4] has performed a perturbation analysis using the energy method and has obtained an expression for attenuation. But this analysis is valid only for very low frequency where attenuation is not very significant [3]. Barker [5] used a viscoelastic analogy to arrive at the attenuation. The equivalent properties are derived from the "mixture theory" [6] which really does not portray the dynamic characteristics of a layered composite. As such, Barker's result for attenuation is independent of frequency and is valid for very high frequency.

There is yet another feature of the dynamic characteristics of a composite which deserves attention. There is experimental evidence [7] of development of tensile forces and subsequent debonding of dynamically compressively loaded composites. The existing analysis [8] based on "transmission and reflection coefficients" again points to a frequency-independent behaviour which is a contradiction to reality.

In the analysis of stress wave propagation in a composite structure one should consider the finite extent of the media, multiple reflections and physically realizable boundary conditions. 
The present method of analysis [3], based on the transfer matrix method, is applicable to finite dimensional structures, does not depend on the Floquet theory and can be applied to any kind of loading. In this paper results are presented for attenuation and maximum tensile stresses developed in periodically layered elastic composites subjected to rectangular compressive pulses.

\section{THEORY}

The composite is assumed to be a periodic arrangement of layers of two homogeneous elastic materials (see Figure 1). An assembly constituted of one layer of each of the

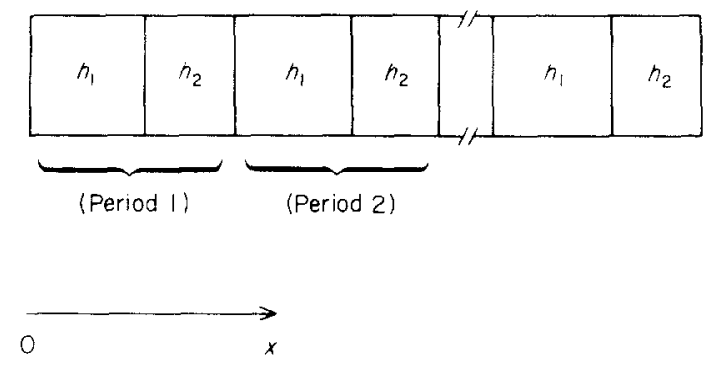

Figure 1. Description of the composite and co-ordinate system.

constituents is termed a period. The analysis is performed within the framework of one space dimension. The equation of motion for the $i$ th layer is

$$
\partial^{2} u_{i} / \partial t^{2}=c_{i}^{2}\left(\partial^{2} u_{i} / \partial x^{2}\right), \quad i=1,2
$$

$c_{i}$ is the speed of the longitudinal wave in the $i$ th layer. $u_{i}(x, t)$ denotes the displacement field within the $i$ th layer. Laplace transforms are taken, for zero initial conditions, and the two equations (1) are solved for the transformed displacement $U_{i}(x, s)$ :

$$
U_{i}(x, s)=\int_{0}^{\infty} \mathrm{e}^{-s t} u_{i}(x, t) \mathrm{d} t
$$

The transform of the stress is obtained as

$$
F_{i}(x, s)=E_{i}\left(\mathrm{~d} U_{i} / \mathrm{d} x\right) .
$$

The four constants of integration from the solution of the transformed equations (1) are eliminated by using the displacements and stresses at the two ends of a period, and by virtue of the continuity of these parameters at the interface of the two layers within the period. This yields the basic transfer matrix for one period relating the transformed displacements and stresses at the two ends of a period [3]. Thus, for the $j$ th period

$$
\left[\begin{array}{c}
U_{j, R} \\
F_{j, R}
\end{array}\right]=[T]\left[\begin{array}{c}
U_{j, L} \\
F_{j, L}
\end{array}\right] .
$$

Subscripts $R$ and $L$ refer to the conditions at the right and left ends of the period, respectively. So, for a $n$-period composite where the alternate layers are identical,

$$
\left[\begin{array}{c}
U_{n, R} \\
F_{n, R}
\end{array}\right]=[T]^{n}\left[\begin{array}{c}
U_{1, L} \\
F_{1, L}
\end{array}\right] .
$$




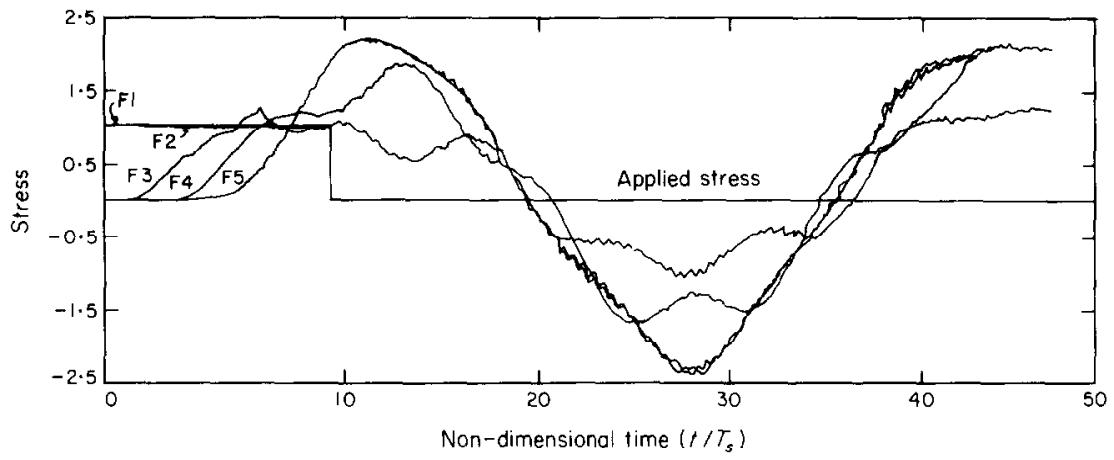

Figure 2. Pulse input response of a four period elastic composite. Pulse width $\left(T_{w} / T_{s}\right)=10$. Material properties: $h_{1}=h_{2}=10 \mathrm{~cm} ; E_{1}=2 \times 10^{6} \mathrm{~kg} / \mathrm{cm}^{2} ; E_{2}=3 \times 10^{4} \mathrm{~kg} / \mathrm{cm}^{2} ; \rho_{1}=7.8 \mathrm{~g} / \mathrm{cm}^{3} ; \rho_{2}=1.1 \mathrm{~g} / \mathrm{cm}^{3}$.

TABLE 1

Material properties of the constituent layers

\begin{tabular}{|c|c|c|c|c|c|}
\hline \multicolumn{2}{|c|}{$\begin{array}{l}\text { Layer thickness } \\
\text { (cm) }\end{array}$} & \multicolumn{2}{|c|}{$\begin{array}{l}\text { Young's modulus } \\
\left(\mathrm{kg} / \mathrm{cm}^{2}\right)\end{array}$} & \multicolumn{2}{|c|}{$\begin{array}{l}\text { Density } \\
\left(\mathrm{g} / \mathrm{cm}^{3}\right)\end{array}$} \\
\hline$h_{1}$ & $h_{2}$ & $E_{1}$ & $E_{2}$ & $\rho_{1}$ & $\rho_{2}$ \\
\hline $10 \cdot 0$ & $10 \cdot 0$ & $2.0 \times 10^{6}$ & $3.0 \times 10^{4}$ & $7 \cdot 8$ & $1 \cdot 1$ \\
\hline
\end{tabular}

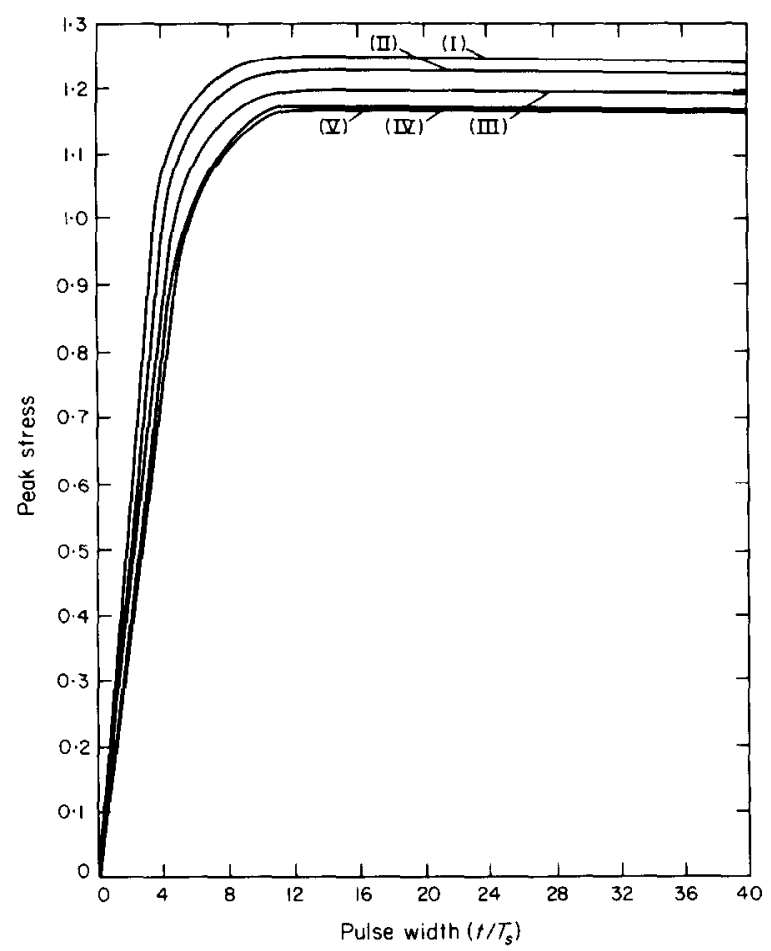

Figure 3. Peak positive stress at various locations in a 13 period elastic composite. First passage response to a unit stress pulse. (i) After 1 period; (ii) after 2 periods; (iii) after 4 periods; (iv) after 8 periods; (v) after 12 periods. 


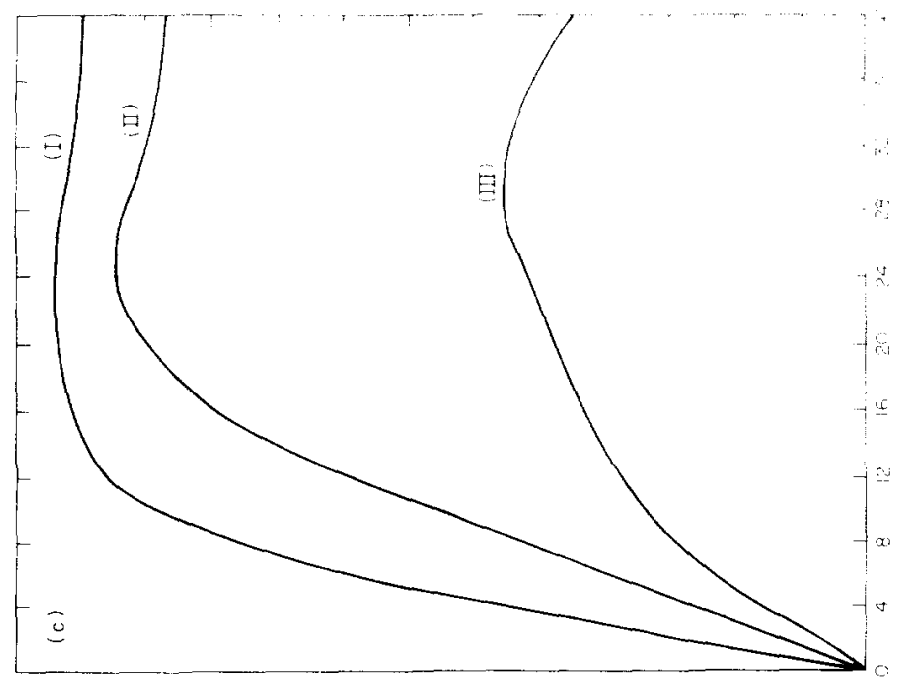

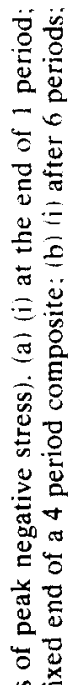

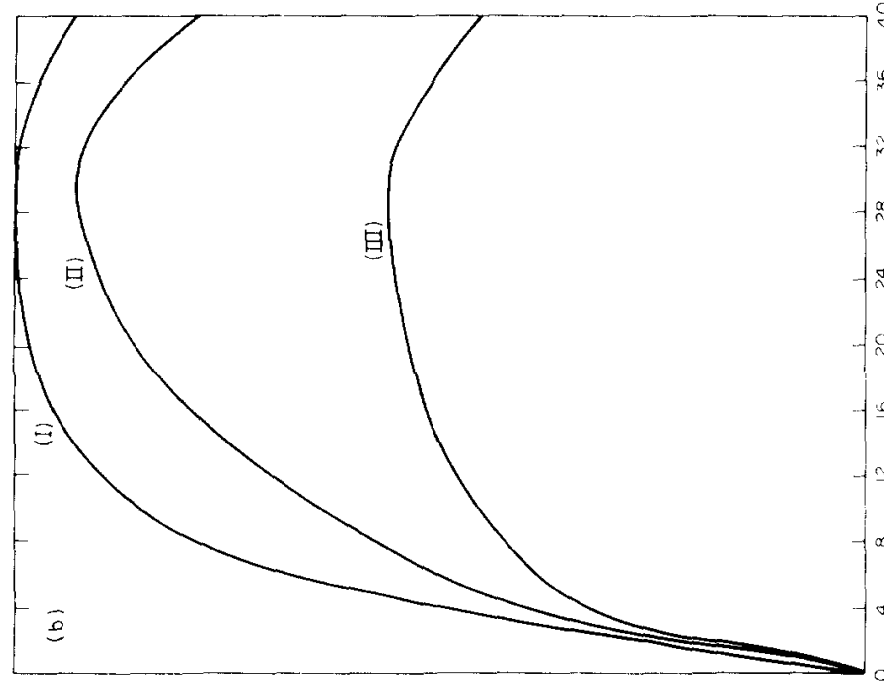

苞焉

$=$

品已

F

却

흘 응

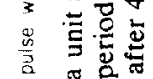

응 웡

$\stackrel{\pi}{\frac{\pi}{m}}$.

要

点

प्टे

影

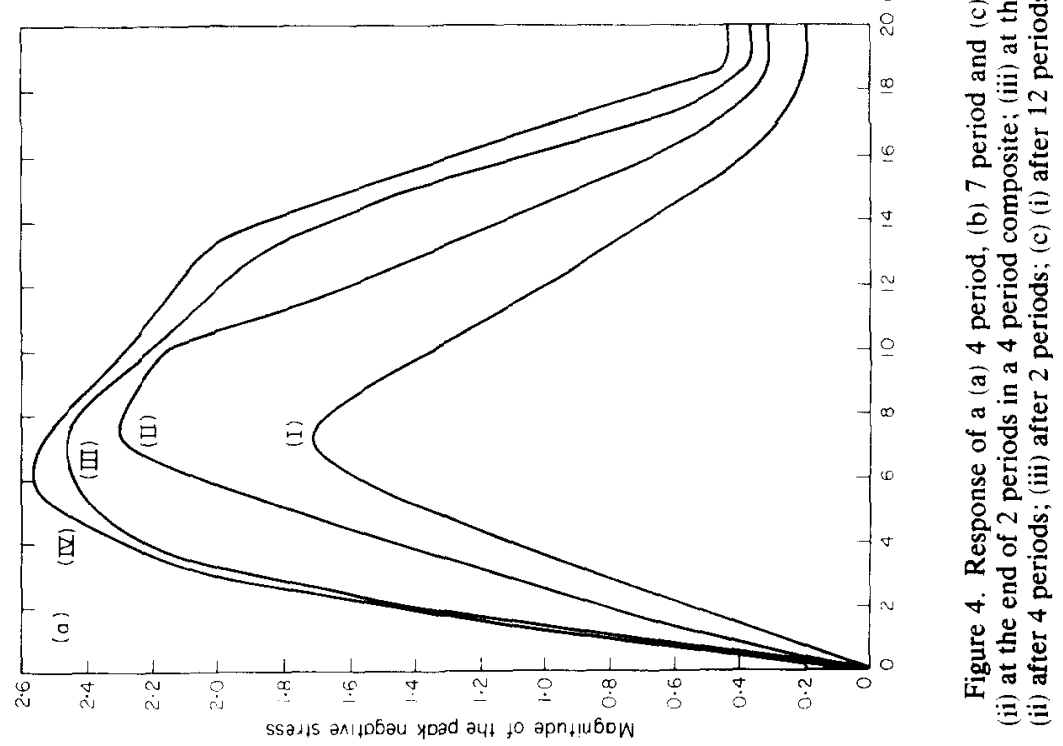


For any intermediate location at the end of the jth period, equation (5) can be used with $j$ replacing $n .[T]^{n}$ is the overall transfer matrix for the entire composite. Boundary conditions in the form of transforms of known displacement or stresses are applied and the final time domain solution is obtained by inversion of the Laplace transform.

\section{NUMERICAL ANALYSIS}

In the numerical examples presented here the composite is assumed to be fixed at the left end: i.e., $U_{1, L}=0$. It is assumed to be excited at the right end by a stress

$$
f_{n, R}=\left\{\begin{array}{ll}
1, & 0<t<T_{w} \\
0, & t>T_{w}
\end{array}\right\} .
$$

One typical response of a composite is presented in Figure 2. Corresponding materal properties are shown in Table 1. It is observed that the pulse is restructured and attenuated in its passage through the composite. Further, a stress of an opposite sign, i.e., a tensile stress, is developed on application of a compressive stress.

A parametric study has been carried out for composites with varying number of periods subjected to unit amplitude stress pulses of varying pulse width, $T_{w}$. Results for peak positive and negative stress are presented in Figures 3 and $4(a)-(c)$. Time has been non-dimensionalized with respect to $T_{s}$, defined as $T_{s}=\left(h_{1} / c_{1}\right)+\left(h_{2} / c_{2}\right)$, where $h_{1}$ and $h_{2}$ are the thicknesses of the constituent layers. For comparison, the attenuation at various locations of a thirteen period composite as predicted by the method of reference [5] is presented in Table 2. As mentioned before, these results are independent of the pulse width. Comparison with Figure 2 shows considerable differences.

\section{DISCUSSION}

Restructuring of the pulse is due to dispersion: i.e., different frequency components of the pulse move with different velocities and arrive at a point at different instants. Negative stress is also due to the phase difference between various frequency components in the incident and reflected stress waves.

Contrary to the predictions of other theories, the attenuation is not uniform in all the layers; rather it is more significant in the first few layers. Again, the attenuation is found to be dependent on the pulse width. This explains the differences in attenuation shown in Figure 2 (present theory) and Table 2 (reference [5]). The magnitude of the peak negative stress, too, is dependent on the pulse width and approaches the value corresponding to that in step loading as the pulse width increases. For shorter pulses it is expectedly small because of greater attenuation. It shows a maximum for some intermediate value of the pulse width. This negative stress, if sufficiently high, will cause delamination of the composite and in design one must guard against this failure.

\section{TABLE 2}

Attenuation predicted by the method of reference [5] for a 13 period composite with material properties as in Table 1 (these results are independent of the pulse width)

\begin{tabular}{cccccc}
\hline & \multicolumn{5}{c}{ Location: End of period } \\
\cline { 2 - 6 } & 1 & 2 & 4 & 8 & 12 \\
\hline Attenuation & $1.68 \times 10^{-1}$ & $2.83 \times 10^{-2}$ & $8.00 \times 10^{-4}$ & $6.40 \times 10^{-7}$ & $5.12 \times 10^{-10}$ \\
\hline
\end{tabular}




\section{REFERENCES}

1. C. SVE 1972 Journal of Applied Mechanics 39,1151-1154. Stress wave attenuation in composite materials.

2. I. MUKONIKI and T.C. T. TING 1980 International Journal of Solids and Structures 16, 239-251. Transient wave propagation normal to the layering of a finite layered medium.

3. A. K. GHOSH 1981 Ph.D Thesis, Indian Institute of Technology, Bombay. Dynamics of periodically layered media.

4. R. M. CHRISTENSEN 1973 Journal of Applied Mechanics 40, 155-160. Attenuation of harmonic waves in layered media.

5. L. M. BARKER 1971 Journal of Composite Materials 5, 140-162. A model for stress wave propagation in composite materials.

6. L. M. BREKHSKOVIKH 1960 Waves in Layered Media. New York: Academic Press.

7. J. D. ACHENBACH, J. H. HemanN and F. ZIEGLER 1968 American Institute of Aeronautics and Astronautics Journal 6, 2040-2043. Tensile failure of interface bonds in a composite body subjected to compressive loads.

8. E. M. DEJAEGAR and H. LEVINE 1969 Applied Science Research 21, 87-91. Reflection and transmission of plane waves by periodically layered media. 